\title{
APTIDÃO FÍSICA DOS ATLETAS DA EQUIPE DO UNIVERSITÁRIO RUGBY SANTA MARIA (URSM) NO PERÍODO PÓS-COMPETITIVO ANO DE 2012
}

Leandro Lima Borges, Universidade Federal de Santa Maria - UFSM, Santa Maria, Rio Grande do Sul-Brasil

Leonardo Fernandes de Souza, Universidade Federal de Santa Maria - UFSM, Santa

Maria, Rio Grande do Sul - Brasil

Ana Paula Ziegler Vey, Universidade Federal de Santa Maria - UFSM, Santa Maria, Rio

Grande do Sul-Brasil

Ana Carolina Vale Fernandes, Universidade Federal de Santa Maria - UFSM, Santa

Maria, Rio Grande do Sul - Brasil

Andressa Ferreira da Silva, Universidade Federal de Santa Maria - UFSM, Santa Maria, Rio Grande do Sul - Brasil

Luciane Sanchotene Etchepare Daronco, Universidade Federal de Santa Maria - UFSM, Santa Maria, Rio Grande do Sul-Brasil

Felipe Flores Guilardi, Universidade Federal de Santa Maria - UFSM, Santa Maria, Rio Grande do Sul-Brasil

\section{RESUMO}

O Rúgbi é um esporte que surgiu na Inglaterra, no ano de 1823, através de uma jogada irregular em uma partida de futebol. Atualmente, esse esporte está entre os que mais crescem, e também estará presente nas Olimpíadas no Rio de Janeiro, em 2016. Porém, apesar do seu crescimento exponencial, ainda é grande o número de pessoas que não possuem maiores conhecimentos sobre este esporte. Este trabalho apresenta um estudo que buscou avaliar a aptidão física dos jogadores de rúgbi da equipe do Universitário Rugby Santa Maria, no período pós-competitivo da temporada 2012. Foram coletados dados de 10 atletas, todos do sexo masculino, com média de idade de 21,4 anos, e foram analisados, de acordo com padrões definidos por autores da área de Medidas e Avaliação. A Resistência Muscular Localizada foi avaliada através do teste de flexão de tronco em 1 minuto, a Resistência Anaeróbia através do teste de corrida de 50 metros e a Resistência Aeróbia através do teste de Cooper. Também foram coletadas as medidas de cintura e quadril (Relação Cintura-Quadril). Os dados foram analisados utilizando estatística descritiva (média).

Palavras-Chave: Rúgbi; Aptidão física; Jogadores.

\section{FITNESS ATHLETES OF UNIVERSITY RUGBY TEAM SANTA MARIA (URSM) IN THE POST-COMPETITION YEAR 2012}

\footnotetext{
ABSTRACT

The Rugby is a sport that originated in England in the year 1823, through an irregular play in a football match. Currently, this sport is among the fastest growing, and will also be present at the Olympics in Rio de Janeiro in 2016. However, despite its exponential 
growth, there is a huge number of people who do not have much knowledge about this sport. This paper presents a study that sought to evaluate the fitness of rugby players team University Rugby Santa Maria, in the post-competitive 2012 season. Data were collected from 10 athletes, all male, with a mean age of 21.4 years and were analyzed according to standards set by authors in the field of measurement and evaluation. The Local Muscular Endurance was assessed by testing for trunk flexion in 1 minute, Anaerobic Endurance by testing 50-meter sprint Aerobic and Resistance through the Cooper test. Were also collected measures of waist and hip circumference (Waist-Hip Ratio). Data were analyzed using descriptive statistics (mean).

Key-Words: Rugby; Physical fitness; Players.

\section{ATLETAS DE ADECUACIÓN DE LA UNIVERSIDAD DE RUGBY DEL EQUIPO DE SANTA MARIA (URSM) EN EI Post- COMPETITION AÑO 2012}

\section{RESUMEN}

El rugby es un deporte que se originó en Inglaterra en el año 1823, a través de un juego irregular en un partido de fútbol. En la actualidad, este deporte es uno de los de más rápido crecimiento, y también estará presente en los Juegos Olímpicos de Río de Janeiro en 2016. Sin embargo, a pesar de su crecimiento exponencial, hay un gran número de personas que no tienen mucho conocimiento sobre este deporte. En este trabajo se presenta un estudio que pretendía evaluar la condición física de los jugadores de rugby equipo de la Universidad de Rugby Santa María, en la época posterior a la competencia 2012. Se recogieron datos de 10 atletas, todos hombres, con una edad media de 21,4 años, y se analizaron de acuerdo a las normas establecidas por los autores en el campo de la medición y la evaluación. La resistencia muscular local se evaluó mediante la prueba de flexión de tronco en 1 minuto, la resistencia anaeróbica por la prueba de 50 metros lisos aeróbico y de resistencia a través de la prueba de Cooper. También se han recogido las medidas de cintura y la circunferencia de la cadera (índice cintura-cadera). Los datos fueron analizados utilizando estadística descriptiva (media).

Palabras-Clave: Rugby; Buena salud; Jugadores. 


\section{INTRODUÇÃO}

Em pesquisa recente realizada no Brasil, o rúgbi foi apontado como o esporte que mais irá crescer nos próximos anos. ${ }^{1}$ Segundo este autor, a pesquisa mostra que apenas $1 \%$ dos entrevistados pratica o rúgbi e somente $3 \%$ responderam que esta é sua modalidade favorita. Além disso, o rúgbi foi apontado como o esporte menos conhecido pelos entrevistados (26\%), seguido por beisebol (20\%) e golfe (20\%). Tais resultados sugerem que há um vasto campo disponível para desenvolver atividades relacionadas a esta modalidade no Brasil.

No Brasil, o rúgbi passa por um processo de crescimento que se reflete nos bons resultados alcançados pelas seleções. A seleção masculina, por exemplo, passou do $45^{\circ}$ lugar para o $27^{\circ}$ no ranking mundial num período de três anos, enquanto a feminina atingiu a $10^{\mathrm{a}}$ posição. $^{2}$

Sumariamente, o rúgbi é um jogo de invasão e evasão, no qual o time que detém a posse da bola tem o objetivo de avançar com ela, carregando-a ou chutando-a, tomando território da equipe adversária e finalmente pontuando. Por sua vez, a equipe sem a posse da bola, tem a tarefa de tenta evitar este avanço.

O rúgbi é um esporte que destaca-se dos demais por vários motivos, mas o que chama a atenção é a paixão com que os praticantes referem-se a esse esporte. Possivelmente, muito desse fervor baseia-se na correspondência entre os princípios utilizados no jogo e que devem ser aplicados aos demais campos da vida cotidiana. No rúgbi, além das 'regras do jogo' existe também o 'espírito das leis', que é um conjunto de princípios que norteia a conduta dos jogadores durante a partida. São eles: integridade, paixão, solidariedade, disciplina e respeito.

Logo, além de formar atletas, o rúgbi ajuda a formar cidadãos dotados de elevado caráter moral. Ao transmitir valores como união, espírito de equipe, solidariedade, lealdade, garra, superação, entrega, educação, respeito ao adversário e aos árbitros, a importância da coletividade sobrepõe-se à individualidade. Por essas razões, considera-se o rúgbi um forte instrumento de desenvolvimento social. 
Nesse sentido, objetivou-se com este estudo verificar a aptidão física dos atletas do Universitário Rugby Santa Maria no período pós-competitivo.

\title{
METODOLOGIA
}

A presente pesquisa caracterizou-se como uma pesquisa diagnóstica exploratória, pois visou diagnosticar uma realidade e explorar os achados através de possíveis relações entre os resultados encontrados com a literatura existente sobre o tema.

\begin{abstract}
Amostra
O estudo foi composto por 10 atletas da equipe masculina do Universitário Rugby Santa Maria. A média de idade dos indivíduos testados é de 21,4 anos. Utilizou-se como critério de exclusão do estudo aqueles atletas que não completaram todos os testes, independente do motivo.
\end{abstract}

\section{Procedimentos}

Foram realizados apenas testes de campo. Os testes realizados foram os seguintes: mensurações das circunferências da cintura, abdome e quadril, teste dos 12 minutos (Cooper), ${ }^{5}$ corrida de 50 metros e teste de flexão de tronco em um minuto. Foi previamente esclarecido que os atletas poderiam encerrar os testes a qualquer momento, devido a qualquer sintoma que poderiam vir a sentir. Foi necessário apenas um dia, ocupando apenas o turno da tarde. A ordem foi a seguinte: mensuração das circunferências da cintura, quadril e abdômen, teste de flexão de tronco em 1 minuto, teste de corrida de 50 metros e teste dos 12 minutos (Cooper). ${ }^{5}$ Após coletados, os dados foram analisados e classificados segundo normas de classificação encontradas na literatura.

Nenhum atleta recebeu qualquer remuneração pela sua participação nos testes.

Foram utilizados como instrumentos da coleta de dados:

- Mensuração das medidas da cintura, abdome e quadril (fita métrica ISP) - para avaliar os riscos de doenças cardiocirculatórias;

- "Teste de abdominal modificado" - para avaliar a resistência muscular localizada;

- "Teste de corrida de 50 metros" - para avaliar a resistência anaeróbica; 
- "Teste dos 12 minutos" (Cooper) ${ }^{5}$ - para avaliar a resistência aeróbica.

\section{Análise estatística dos dados}

Foi utilizada estatística descritiva para verificar média dos dados coletados.

\section{RESULTADOS E DISCUSSÃO}

A análise e discussão dos resultados deram-se da seguinte forma:

- tabela com os resultados obtidos;

- discussão dos resultados considerados abaixo da média segundo normas de classificação;

- discussão dos resultados considerados na média ou acima da média segundo normas de classificação.

Tabela 1 - Resultados obtidos pela equipe do URSM, média

\begin{tabular}{lc}
\hline Variáveis & Média \\
\hline Idade (anos) & 21,4 \\
RCQ & 0,84 \\
Cooper (ml/kg/min) & 36,43 \\
50 Metros (s) & 7,86 \\
Abdominal (repetições) & 42,1 \\
\hline
\end{tabular}

Foram realizadas avaliações com 13 atletas da equipe do Universitário Rugby Santa Maria, mas somente 10 completaram as avaliações. Pode-se perceber na Tabela 1 que o grupo possui uma média de idade de 21,4 anos, considerada bastante jovem.

A Tabela 1 proporciona também a análise das seguintes variáveis: Relação Cintura Quadril - RCQ, Resistência Muscular Localizada - RML (abdominal em 1 minuto), Resistência Cardiorrespiratória - RCR (Cooper) e Resistência Anaeróbia (Corrida de 50 metros).

Sobre a resistência aeróbia, em estudo realizado com jogadores de futebol profissionais, juniores e juvenis, através do teste de Léger ${ }^{4}$ - que caracteriza-se por repetidas corridas de 
20 metros, com períodos de recuperação e que a velocidade vai aumentando através de sinais sonoros -, valores 50,21, 49,58 e 49,53 .ml (kg.min) ${ }^{-1}$ (respectivamente profissionais, juniores e juvenis). ${ }^{3}$ Percebe-se que são valores bastante superiores aos encontrados no teste realizado com a equipe do URSM, que na Tabela 2 encontram-se no nível "Fraco".

Tabela 2 - Valores de referencias de VO2máx. ml (kg.min $)^{-1}$ - homens

\begin{tabular}{ccccccc}
\hline Idade & Muito Fraca & Fraca & Regular & Boa & Excelente & Superior \\
\hline $13-19$ & $-35,0$ & 35,1 a 38,3 & 38,4 a 45,1 & 45,2 a 50,9 & 51,0 a 55,9 & $>56,0$ \\
$20-29$ & $-33,0$ & 33,1 a 36,4 & 36,5 a 42,4 & 42,5 a 46,4 & 46,5 a 52,4 & $>52,5$ \\
$30-39$ & $-31,5$ & 31,6 a 35,4 & 35,5 a 40,9 & 41,0 a 44,9 & 45,0 a 49,4 & $>49,5$ \\
$40-49$ & $-30,2$ & 30,3 a 33,5 & 33,6 a 38,9 & 39,0 a 43,7 & 43,8 a 48,0 & $>48,1$ \\
$50-59$ & $-26,1$ & 26,2 a 30,9 & 31,0 a 35,7 & 35,8 a 40,9 & 41,0 a 45,3 & $>45,4$ \\
Mais de 60 & $-20,5$ & 20,6 a 26,0 & 26,1 a 32,3 & 32,3 a 36,4 & 36,5 a 44,2 & $>44,3$ \\
\hline
\end{tabular}

Fonte $^{5}$

A Resistência Anaeróbia obteve um resultado considerado fraco: na corrida de 50 metros a média foi superior a 7 segundos $(7,86)$. Segundo a tabela 3 de classificação citada ${ }^{6}$, é um índice considerado 'fraco'.

Poucos estudos utilizando o teste de corrida de 50 metros foram encontrados, sendo que a maioria foram de sprints de 30 à 40 metros e com jogadores de futebol. Em estudo realizado com atletas de uma escola de futebol de campo de Lages/SC utilizando o teste de corrida 50 metros $^{7}$, em que buscaram verificar a velocidade de deslocamento. ${ }^{8}$ Pode-se perceber que em virtude da média de idade de 15,03 \pm 1,45 anos os tempos foram altos (uma média de 7,84 segundos), sendo valores semelhantes com os encontrados com os atletas do URSM. Segundo a literatura, a corrida de 50 metros é um dos métodos mais utilizados para medir de maneira indireta a potência anaeróbia alática, pois os 50 metros são percorridos em torno de 10 segundos (pico máximo de metabolismo ATP-CP). ${ }^{9}$

Conforme alguns autores, grande parte da capacidade de velocidade é determinada pela genética, mas, apesar da forte relação da velocidade com a genética, ela não é um fator limitante, pois os atletas podem melhorar a sua capacidade com o treinamento. ${ }^{10} \mathrm{Em}$ estudo que visou identificar o perfil morfofuncional dos jogadores de rúgbi juniores e sub- 
21 da seleção nacional de Portugal, com média de idade de 18 anos, foi realizado os testes de RML (abdominais 40'), resistência anaeróbia (corrida de 50 metros) e resistência cardiorrespiratória (Progressive Shuttle Run Test form Multistage Fitness Test, 20m). ${ }^{11-12}$ Para o teste de velocidade (avançados e linha atrasada), o mesmo autor encontrou os valores 7,08 \pm 0,21 segundos (avançados) e 6,86 \pm 0,20 segundos (linha atrasada), respectivamente, sendo valores próximos ao encontrado no estudo com a equipe do URSM (valores estes classificados como fracos a partir das normas de classificação da TABELA $3)^{6}$

Tabela 3 - Classificação do Teste de Corrida 50m (Futebol)

\begin{tabular}{cc}
\hline Índice alcançado (T em segundos) & Classificação \\
\hline $5-5,5$ & Excelente \\
$5,6-6 \mathrm{~s}$ & Muito Bom \\
$6,1-6,5$ & Bom \\
$6,6-7 \mathrm{~s}$ & Médio \\
$>7$ & Fraco \\
\hline Fonte $^{6}$ &
\end{tabular}

Na variável Resistência Muscular Localizada e Relação Cintura-Quadril, os resultados foram considerados bons segundo algumas relevantes normas de classificação (TABELAS 4,5 respectivamente). ${ }^{13-16}$

Em estudo, que tinha como objetivo de identificar o perfil antropométrico e o desempenho motor de atletas de futsal masculino das equipes finalistas do campeonato paranaense da categoria adulto, avaliou a resistência muscular localizada através do teste de abdominal em 1 minuto. ${ }^{14}$ Os atletas deste estudo apresentaram uma média de 55 repetições, superior ao número de repetições realizadas pela equipe do URSM que foi de 42,1. Na Tabela 4, a equipe do URSM se encontra no nível de classificação "médio". 
Tabela 4 - Normas para Teste Abdominal 1 minuto - masculino

\begin{tabular}{cccccc}
\hline Idade & Excelente & Bom & Médio & Regular & Fraco \\
\hline $20-29$ & $>48$ & $43-47$ & $37-42$ & $33-36$ & $0-32$ \\
$30-39$ & $40-47$ & $35-39$ & $29-34$ & $25-28$ & $0-24$ \\
$40-49$ & $35-39$ & $30-34$ & $24-29$ & $20-23$ & $0-19$ \\
$50-59$ & $30-34$ & $25-29$ & $19-24$ & $15-18$ & $0-4$ \\
$>60$ & $25-29$ & $20-24$ & $14-19$ & $10-13$ & $0-9$ \\
\hline Fonte $^{13}$ & & & & &
\end{tabular}

Através da literatura, ainda verificou-se que em estudo realizado com atletas da equipe de handebol campeã dos jogos municipais da cidade de Ponta Grossa/PR, em que foi avaliada a resistência muscular localizada, obtendo uma média de 43,64, semelhante ao número de repetições realizadas pelos atletas do URSM. ${ }^{15}$

Nesse estudo, uma das variáveis avaliadas na parte da composição corporal foi a da Relação Cintura Quadril. Tal equipe obteve o valor médio de 0,83 , resultado também semelhante a equipe do URSM $(0,84)$ - considerado "moderado" segundo as normas de classificação. ${ }^{16}$

Tabela 5 - Normas para classificação da PCCQ - Proporção Circunferência CinturaQuadril (Homens)

\begin{tabular}{ccccc}
\hline Idade & Baixo & Moderado & Alto & Muito Alto \\
\hline Até 29 & $<0,83$ & $0,83-0,88$ & $0,89-0,94$ & $>0,94$ \\
$30-39$ & $<0,84$ & $0,84-0,91$ & $0,92-0,96$ & $>0,96$ \\
$40-49$ & $<0,88$ & $0,88-0,95$ & $0,96-1,00$ & $>1,00$ \\
$50-59$ & $<0,90$ & $0,90-0,96$ & $0,97-1,02$ & $>1,02$ \\
$60-69$ & $<0,91$ & $0,91-0,98$ & $0,99-1,03$ & $>1,03$ \\
\hline Fonte $^{16}$ & & & &
\end{tabular}

Atualmente, a relação cintura quadril trata-se de uma ferramenta paralela, rápida, fácil e muito eficiente para identificar pessoas com risco de saúde elevado devido à gordura abdominal. ${ }^{17}$ Ainda, segundo a literatura existente, apenas a medida da cintura já seria 
suficiente para avaliar a presença de depósito de gordura visceral comparado à RCQ, pois ao apresentar um valor $>89 \mathrm{~cm}$ para mulheres e $>101 \mathrm{~cm}$ para homens por si só já seria um sinal de risco. ${ }^{18-19}$

Além do mais, o acúmulo de gordura intra-abdominal se torna prejudicial quando o indivíduo se submete a um estresse muito alto, pois essa gordura desprende da cavidade intra-abdominal e pode acarretar desordens metabólicas, aumentando assim os riscos de doenças cardiovasculares. Como a medida de RCQ revela a distribuição da gordura no indivíduo, ela é um fator importante para verificar onde há maior localização de gordura e conforme afirma ainda o autor, quanto maior a quantidade de gordura abdominal, maior o risco de doenças cardiovasculares. ${ }^{20}$

\section{CONCLUSÃO}

Através deste trabalho pode-se ver que, de modo geral, a classificação dos níveis de aptidão física estão razoavelmente aquém do esperado de uma equipe competitiva. Ao mesmo tempo em que obtiveram resultados satisfatórios na avaliação da resistência muscular localizada e se encontram dentro da classificação moderada para a relação cintura-quadril, obtiveram resultados insatisfatórios nas avaliações da resistência anaeróbia e aeróbica.

O presente estudo trouxe benefícios a respeito do tema abordado, contribuindo na verificação de um perfil físico dos atletas do Universitário Rugby Santa Maria, servindo como diagnóstico e parâmetro para prescrição de uma preparação física adequada, enfatizando as qualidades físicas deficitárias.

Sugere-se que estes mesmos testes sejam realizados posteriormente para efeito de comparação, de modo a indicar se o treinamento utilizado pela equipe do URSM se mostra eficiente ao longo da temporada.

\section{REFERÊNCIAS}

1DELOITTE, T. T. Muito além do futebol: estudo sobre esporte no Brasil, 2011. Disponível em: $\quad$ http://www.deloitte.com/assets/Dcom- 
Brazil/Local\%20Assets/Documents/Estudos\%20e\%20pesquisas/PesquisaMuitoAlemFuteb ol.pdf>. Acesso em: 12 mar. 2012.

${ }^{2}$ CONFEDERAÇÃO BRASILEIRA DE RUGBY. Disponível em: <http://www.brasilrugby.com.br/>. Acesso em: 3 maio 2012.

${ }^{3}$ CAMPEIZ, J. M.; OLIVEIRA, P. R.; MAIA, G. B. M. Análise de variáveis aeróbias e antropométricas de futebolistas profissionais, juniores e juvenis. Conexões: revista da Faculdade de Educação Física da UNICAMP, Campinas, v. 2, n.1, 2004.

${ }^{4}$ LÉGER, L. A.; LAMBERT, J. A maximal multistage 20-m shuttle run test to predict V02max. European Journal of Applied Physiology, Heidelberger, v. 49, 1982.

${ }^{5}$ COOPER, K. H. A means of assessing maximal oxygen uptake. Journal of the American Medical Association, Chicago, v. 203, p. 201-204, 1968.

${ }^{6}$ PITANGA, F. J. G. Testes, medidas e avaliação em educação física e esportes. 5. ed. São Paulo: Phorte, 2008.

${ }^{7}$ JOHNSON, B. L.; NELSON, J. K. Practical Measurements for evaluation in physical education. Minessota: Burges, 1979.

${ }^{8}$ FALK, P. R. A.; PEREIRA, D. P. Utilização do teste de 50 metros na mensuração da velocidade em adolescentes de Lages/SC. Publicado em 31 de março de 2009. Disponível em: <http://www.webartigos.com/artigos/utilizacao-do-teste-de-50-metros-na-mensuracaoda-velocidade-em-adolescentes-de-lages-sc/16185/>. Acesso em: 1 jul. 2012.

${ }^{9}$ MATSudo, V. K. R. Testes em ciências do esporte. 4. ed. São Caetano do Sul: CELAFISCS, 1987.

${ }^{10}$ BOMPA, T. Periodização: teoria e metodologia do treinamento. 4. ed. São Paulo: Phorte, 2002. 
${ }^{11}$ VAZ, L. M. T. Perfil morfo-funcional dos jogadores de rugby juniores (Avançados/ Linha Atrasada). Disponível em: <http://www.efdeportes.com/efd40/rugby.htm>. Revista Digital, Buenos Aires, ano 7, n. 40, sept. 2001. Acesso em: 3 jul. 2012.

${ }^{12}$ RAMSBOTTOM, R.; BREWER, J.; WILLIAMS, C. A progressive shuttle run test to estimate maximal oxygen uptake. British Journal of Sports Medicine, Loughboroug, v. 22, p. 141-144, 1988.

${ }^{13}$ POLLOCK, M. L.; WILMORE, J. H. Exercícios na saúde e na doença: avaliação e prescrição para reabilitação. 2. ed. Rio de Janeiro: MEDSI, 1993.

${ }^{14}$ AVELAR, A. et al. Perfil antropométrico e de desempenho motor de atletas paranaenses de futsal de elite. Revista Brasileira de Cineantropometria e Desempenho Humano, Florianópolis, v. 10, n. 1, p. 76-80. 2008.

${ }^{15}$ LEVANDOSKI, G. et al. Composição corporal e aptidão física de atletas de handebol masculino campeões dos XXII jogos estudantis municipais da cidade de Ponta Grossa. Publicatio UEPG: ciências biológicas e da saúde, Ponta Grossa, 14, jul. 2009. Disponível em: <http://www.revistas2.uepg.br/index.php/biologica/article/view/483/484>. Acesso em: 01 jul. 2012.

${ }^{16}$ BRAY, G. A.; GRAY, D. S. Obesity: pathogenesis. Western Journal of Medicine, San Francisco, v.149, p. 429-441, 1988.

${ }^{17}$ BARROW et. al. Medidas e avaliação em educação física e esportes. 5. ed. Barueri: Manole, 2003.

${ }^{18}$ LEAN, M. E. J.; HAN, T. S. Waist circumference as a measure for indicating need for weight management. British Medical Journal, London, v. 311, n. p. 158, 1995.

${ }^{19}$ NATIONAL HEART, LUNG AND BLOOD INSTITUTE. Clinical guidelines on the identification, evaluation, and treatment of overweight and obesity in adults, Washington: National Institutes of Health, 1998. 
${ }^{20}$ AMER, N. M. et al. Índice de massa corporal e razão cintura/quadril de praticantes de atividade aeróbica moderada. Revista da Educação Física/UEM, Maringá, v. 12, n. 2, p. 97-103, 2001.

Recebido em: 05 maio 2013

Aceito em: 13 06. 2013

Contato: Leandro Lima Borges lelimaborges@yahoo.com.br 